\title{
Dark modes engineering in metasurfaces by symmetry matching approach
}

\author{
Elena Bochkova ${ }^{1}$, Shah Nawaz Burokur ${ }^{2}$, André de Lustrac ${ }^{1,3}$ and Anatole Lupu ${ }^{1}$ \\ ${ }^{1}$ Centre de Nanosciences et de Nanotechnologies, CNRS, Univ. Paris-Sud, Université Paris-Saclay, C2N - Orsay, 91405 \\ Orsay, France \\ ${ }^{2}$ LEME, UPL, Univ Paris Nanterre, F92410 Ville d'Avray, France \\ ${ }^{3}$ UPL, Univ Paris Nanterre, F92410 Ville d'Avray, France \\ *corresponding author, E-mail: sburokur@ parisnanterre.fr
}

\begin{abstract}
We revisit the engineering of metasurfaces in order to obtain sharp features in their spectral response. We show that in contrast to conventional approach exploiting indirect mode hybridization mechanism based on strong near-field coupling, a more flexible and efficient engineering of the spectral response can be achieved by using a symmetry matching approach for the excitation of dark modes. This distinctly different mechanism takes advantage of the geometry symmetry of the structure with regard to the incident external electromagnetic field for a direct far-field coupling.
\end{abstract}

\section{Introduction}

The phenomenon of electromagnetically induced transparency (EIT), borrowed from the atomic physics [1], has widely pervaded during the last decade in the fields of photonics [2],[3], plasmonics [4] and metamaterials [5]. The interest in EIT-like resonances is motivated by their ability to display sharp spectral feature that are much narrower as those of uncoupled individual resonators. In the field of plasmonics, such spectral features with steep intensity variation are highly desirable for sensing and switching applications [6]-[8]. The sensing operation is based on detecting a change in plasmon resonance frequency. The change of dielectric environment of the localized surface plasmons induced by the adsorption of the analyte species on the surface of resonant metallic elements results in a shift of their resonance frequency. The sensing detection limit and accuracy are greatly increased by resonances using narrow width spectral features.

Despite the seemingly great variety of studied designs [6], most of them actually share the same operation principle. It consists in associating a superradiant element bearing an electric dipolar momentum and acting as a radiative or bright mode, with a subradiant element bearing an electric quadrupolar or magnetic dipolar momentum and playing the role of a trapped or dark mode [7],[8]. The indirect mode hybridization induced by a strong near-field coupling between bright and dark elements leads to the opening of a narrow transparent window inside the absorption band, called electromagnetically induced transparency (EIT).

The aim of the current contribution is to show that electromagnetically induced transparency and dark mode excitation are not necessary associated. We bring theoretical and experimental evidence that dark mode excitation can be achieved in an independent manner by using direct excitation mechanisms through symmetry matching of the resonant element and that of the incident electromagnetic field. The latter resonance is not related to the coupling of adjacent elements and can be tuned by incidence angle of the external electromagnetic field. By using Babinet's principle, we also demonstrate the validity of this approach for complementary structures configuration [9]-[11]. Thus, narrow dark mode resonances can be achieved in reflection as well as in transmission. The presented approach provides higher flexibility for metasurfaces engineering and results in a great improvement of their spectral performances.

Experimental validation of the proposed symmetry matching approach is performed at microwave frequencies. Two metasurfaces composed of resonant $Z$-shaped metaatoms are designed, fabricated and experimentally tested. Good agreement is found between simulations and measurements.

\section{Direct dark mode excitation through symmetry matching}

Since the initial seminal paper by Fedotov et al. [12], the concept of trapped or dark mode weakly coupled to free space started to be associated with the phenomenon of EIT. However, recent theoretical advances lead to revisit this commonly shared interpretation. In particular it was pointed that no dark mode excitation is necessary for EIT resonances. They can be described by the interference of bright modes only [13]-[15]. In this context, it is natural to wonder whether it is possible to excite a dark mode in a standalone resonant element that is not the result of mode hybridization mechanism between two resonant elements. A 
first answer to this question was given in our recent works [16], [17]. It consists in designing geometry with inversion symmetry that provides matching the induced currents by the incident electric and magnetic fields.

It is well known that the electromagnetic response of metasurfaces is based on surface plasmons which are collective oscillations of free electrons in metal excited by external field. The excited modes are associated to induced dipole moments or currents in the constituting elements. Form of currents is determined by element geometry and its position with respect to incident electromagnetic field. To explain resonance behavior of complex structures through resonator symmetry, group theory approach is often used, particularly for investigating anisotropic optical designs [18]-[21]. However in most simple cases, one can dispense with general considerations.

Let us consider some examples displayed in Fig. 1. Under normal incidence with a vertically polarized electric field $E$, the excitation of a single cut wire (CW) presents an induced electric dipole with currents flowing from one edge to the other (Fig. 1a). A split ring resonator (SRR) without any symmetry with respect to polarization of $E$-field exhibits the same resonance behavior as illustrated in Fig. 1b. Similar result is also observed for $180^{\circ}$ rotated structure except for current flow from clockwise to counterclockwise direction as displayed in Fig.1c. If a structural geometry possesses mirror symmetry with respect to direction of incident $E$-field, the excited currents will be also symmetric as shown in Fig. 1d, since electric field is even, i.e. $E(-\mathrm{r})=-E(\mathrm{r})$. Dark mode resonance is weakly coupled to free space due to vanishingly low projection of dipole moments on incident field. One of possible configurations of dark mode excitation is schematically depicted in Fig. 1e, where two opposite dipole moments are excited. Therefore, the geometry supporting such excitation should not possess mirror symmetry with regard to electric field so as to prevent symmetric and parallel current distributions shown in Fig. 1d.
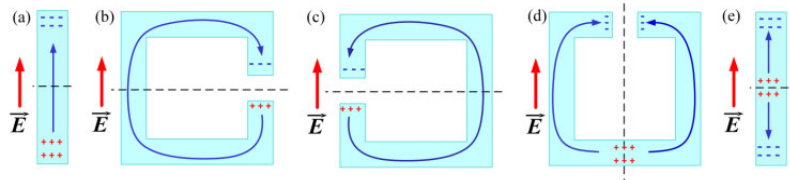

Figure 1: Schematic of external excitation under normal incidence with vertically polarized electric field $E$. (a)-(d) fundamental electric dipole excitation for single $\mathrm{CW}$ and SRR. (e) Schematic representation of forbidden dark mode excitation.

The important symmetry property of a resonator with regard to the magnetic field $H$ must also be emphasized. Owing to axial nature of magnetic field, induced current flows in the same direction for rotated geometry as shown in Fig. 2. Such distinction from electric field behavior will be further used for matching symmetry of the resonator geometry. Using the discussed brief considerations, analysis of the optimal geometry for dark mode excitation is performed.
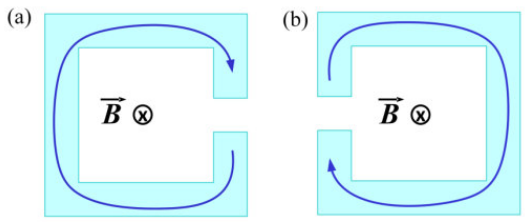

Figure 2: Schematic of induced current by magnetic field for (a) SRR and (b) rotated SRR by $180^{\circ}$.

Since the dimensions of resonators constituting the metasurface are much smaller than wavelength of incident electromagnetic field, induced dipole moment $p$ is linearly dependent on incident electric field according to quasi-static approximation [22]. Thus, the interaction of induced dipole with external $E$-field is more effective when they are parallel. For this reason, both the edges and the center of structure should lie on the same line aligned to the direction of $E$-field. One of the simplest geometry that satisfies all these considerations is presented in Fig. 3, where two antisymmetric $\mathrm{V}$-antennas (AVA) are aligned along $x$-axis. Such AVA structure can be simplified to the $Z$-shaped resonator [16].

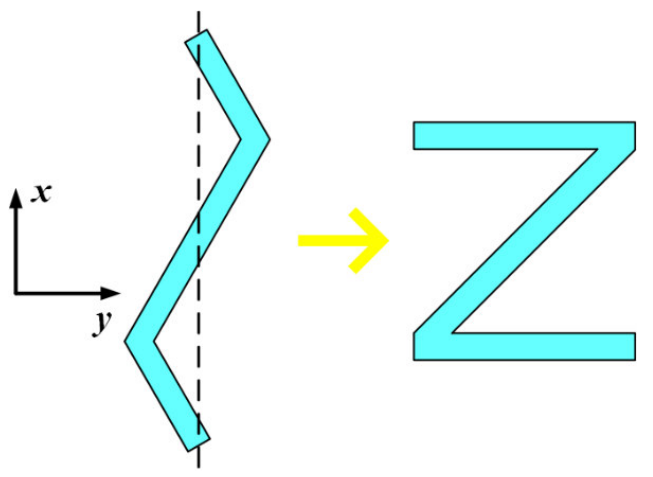

Figure 3: The antisymmetric V-antennas structure presenting no symmetry with respect to vertically aligned $E$-field can be simplified to the $Z$-shaped resonator.

As detailed in [16], dark mode is forbidden under normal incidence and cannot be excited due to zero net electric dipole moment. The mechanism based on using inversion symmetry of the designed resonator for interaction with magnetic field $H$ is used to excite the dark mode resonance. The principle is based on the fact that magnetic field is axial and therefore is invariant to the inversion coordinate transformation as shown in Fig. 2. Hence the magnetic moment induced by opposite currents in the top and bottom $\mathrm{V}$-antennas are oriented in the same direction that leads to net non-zero magnetic dipole moment. Thus, external magnetic field can support dark mode current configuration through direct interaction with external electromagnetic field under oblique incidence. Such approach based on matching the symmetry properties of the resonator with respect to electric and magnetic fields leads to magnetic dipole excitation with reduced electric dipole, allowing to considerably decrease radiation losses and therefore achieving higher quality factor $Q$.

The symmetry matching approach allows to obtain dark mode excitation associated with the first higher order mode 
of odd symmetry which is characterized by two opposite electric dipole moments. This method is demonstrated by considering a metasurface composed of identical Z-shaped resonators as schematically depicted in Fig. 4a. The dielectric substrate used for the metasurface is a single-sided copper-clad epoxy with a relative dielectric constant $\varepsilon_{\mathrm{r}}=3.9$, tangential losses $\tan \delta=0.02$, and a thickness $t=0.4 \mathrm{~mm}$. The period of the unit cell are $p_{\mathrm{x}}=p_{\mathrm{y}}=6 \mathrm{~mm}$, the geometrical dimensions of the $Z$-element along $x$ - and $y$-axis are $l_{\mathrm{x}}=5.8 \mathrm{~mm}$ and $l_{\mathrm{y}}=5.7 \mathrm{~mm}$, respectively, and the width of the metallic wire is $w=0.3 \mathrm{~mm}$. The polarization of electric component of incident electromagnetic field is oriented along $x$-axis. The electromagnetic behavior of $Z$ design is numerically simulated with the finite element method (FEM) of Maxwell's equations using high frequency structure simulator (HFSS) commercial code by ANSYS [23].

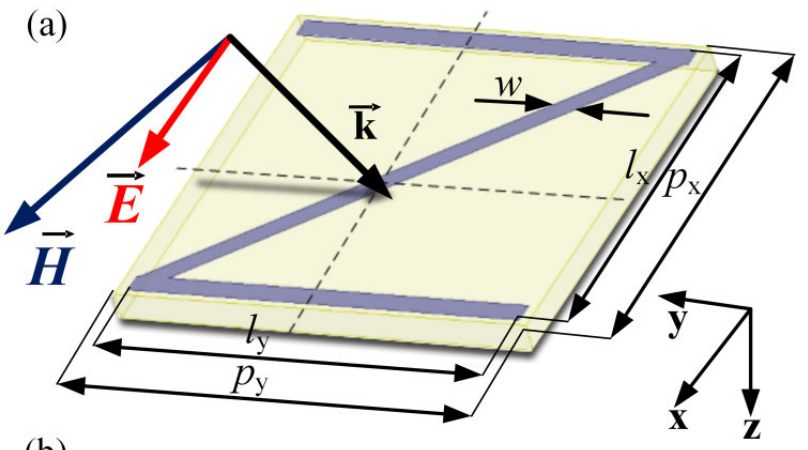

(b)

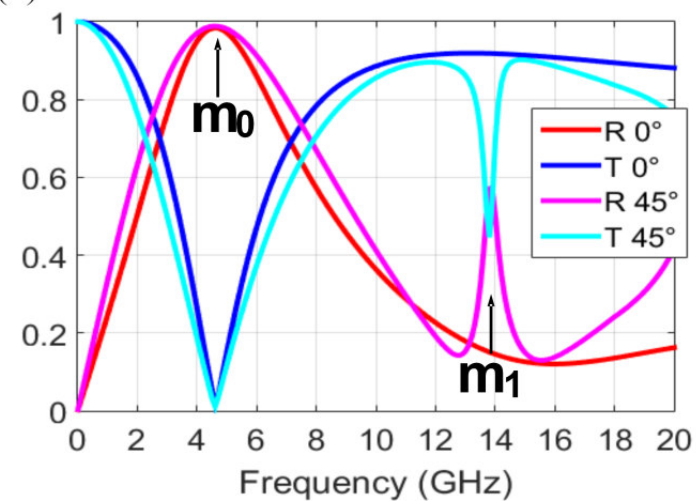

Figure 4: (a) Design of the $Z$-shaped element. Geometrical parameters are $p_{\mathrm{x}}=p_{\mathrm{y}}=6 \mathrm{~mm}, l_{\mathrm{y}}=5.7 \mathrm{~mm}, l_{\mathrm{x}}=5.8 \mathrm{~mm}, w=$ $0.3 \mathrm{~mm}$. (b) Calculated transmission and reflection spectra under normal and oblique incidence.

Under normal incidence when the magnetic field component lies in the plane of the metasurface, only fundamental mode $m_{0}$ is excited at $4.6 \mathrm{GHz}$ as displayed in Fig. 4b. The electric dipole nature of this resonance is confirmed by calculated instantaneous charge distributions as shown in Fig. 5a. The anti-symmetric mode is forbidden due to the zero net electric dipole moment and is not manifested in spectral response. However, dark mode excitation becomes allowed for oblique incidence when the magnetic field interacts with the metasurface. Owing to inversion symmetry of the $Z$ structure, magnetic field induces current loops in opposite directions in the top and bottom parts of the $Z$ structure as depicted in Fig. 5b, such that a magnetic momentum is created. Thus, this dark mode excitation corresponds to that of a magnetic dipole with strongly reduced net electric dipole moment. Hence, significant decrease of radiative losses is observed compared to the electric dipole fundamental mode. The dark mode resonance $\left(m_{1}\right)$ appears at $13.7 \mathrm{GHz}$ and represents a maximum in reflection and minimum in transmission, as it can be clearly observed from spectral characteristics in Figs. $4 \mathrm{~b}, 6 \mathrm{a}$ and $6 \mathrm{c}$.

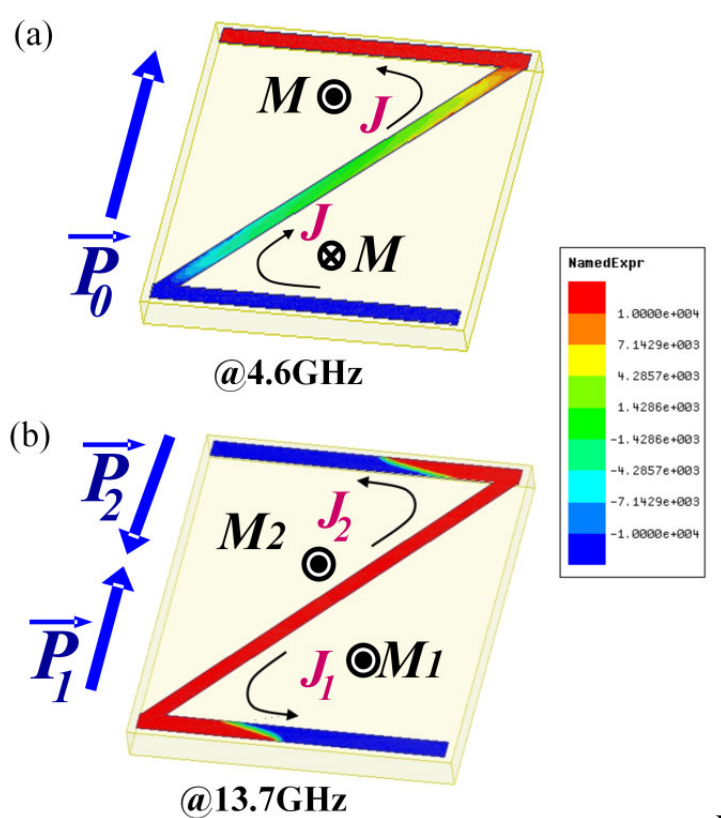

$\mathrm{Fi}$

gure 5: Calculated charge distribution for $Z$-shaped element for (a) fundamental and (b) dark mode resonances. In contrast to normal incidence, dark mode is excited under oblique incidence due to a non-zero net dipolar magnetic momentum, as shown by the charge distribution at 13.7 GHz. Local current flows inducing dipolar momenta are schematically indicated by arrows.

\section{Dark mode engineering in complementary metasurfaces}

In this section, we present the validity of the dark mode excitation by symmetry matching approach applied to complementary designs, i.e. apertures in a metallic sheet. According to Babinet's principle, the complementary structure is introduced by replacing the metallic parts with identical apertures in a metallic sheet and applying duality transformation to the incident field [24]:

$$
\begin{aligned}
& \vec{E}_{c}^{0}=c \vec{B}^{0} \\
& \vec{B}_{c}^{0}=-\frac{1}{c} \vec{E}^{0}
\end{aligned}
$$


where $B^{0}, E^{0}$ and $B_{\mathrm{c}}, E_{\mathrm{c}}$ are the excitation fields for original and complementary case, respectively. The scattering field of the complementary configuration is dual, which in turns results in dual spectral response.

We introduce the complementary configuration for the $Z$ shaped metasurface as presented in the inset of Fig. 6b. As follows from the Babinet's principle, polarization of complementary incident field is such that electric field is horizontally oriented while magnetic field is vertically polarized. As it is expected, the spectral characteristics are inverted for the complementary $Z$ structure, as presented in Figs. $6 \mathrm{~b}$ and $6 \mathrm{~d}$. Therefore, the maximum in reflection as shown in Fig. 6a for the original case (metallic $Z$ structure printed on a dielectric substrate) is replaced by the peak in transmission for both fundamental and dark mode resonances. The slight difference in the positions of resonance frequency and deviation in the line shape can be explained by the presence of dielectric substrate supporting metal sheet in the complementary design [9]. In contrast to the original case, the dark mode is excited at $12.9 \mathrm{GHz}$ under oblique incidence when electric field penetrates through the metasurface. The resonance response increases at rising incident angle due to increase of normal projection of external $E$-field.
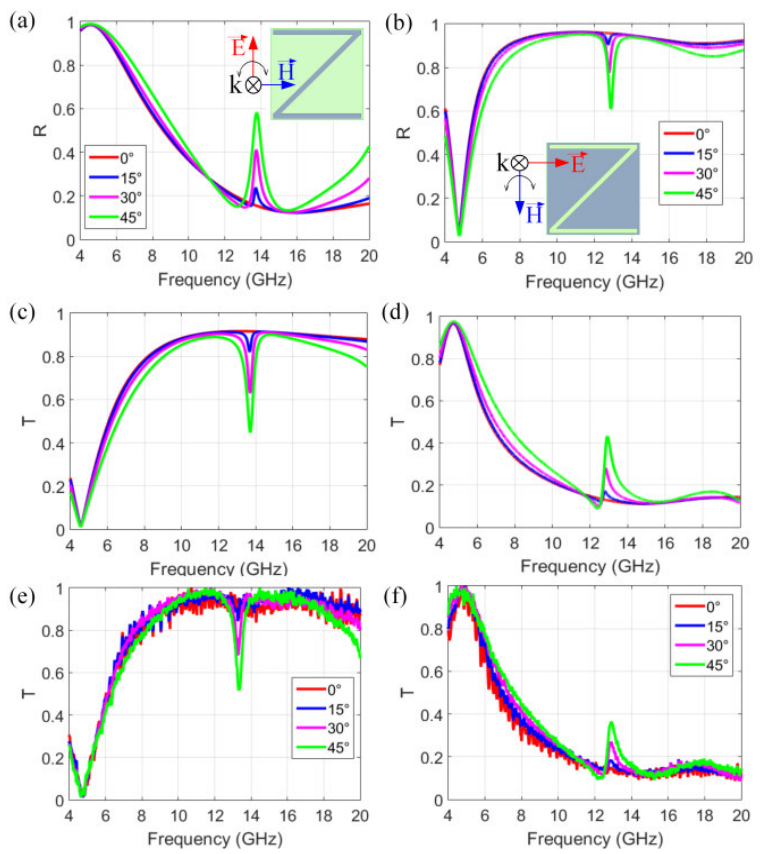

Figure 6: Simulated (a)-(d) and measured (e)-(f) spectral characteristics under normal and oblique incidence for simple (left column) and complementary (right column) Zresonator. A maximum in reflection is obtained for the metallic $Z$ structure printed on a dielectric substrate, while a maximum in transmission is produced by the complementary structure for both fundamental and dark mode resonances.

To understand excitation mechanism, let us consider instantaneous charge distributions for both fundamental and dark mode resonances and demonstrate agreement with analysis provided by Babinet's principle. While the fundamental resonance in the original metallic $Z$ structure is related to the electric dipole excitation along $x$-axis (Fig. $5 a$ ), according to duality the $Z$-shaped aperture produces magnetic dipole excitation along the same direction. This in turn induces an axial electric field as shown in Fig. 7a. The mirror symmetry of the electric lines with respect to the metallic plane relies on the continuity of the normal component of electric field. As it can be observed from Fig. $7 \mathrm{a}$, excited charge distribution where the positive and negative charges are located on the different sides of slot, coincide with the induced electric vectors. In the metallic $Z$ structure, it should be noted that current loops in the top and bottom corners of the $Z$ induce opposite magnetic moments which are orthogonal to the metasurface, as illustrated in Fig. 5a. The dual analogy for the complementary $Z$ represents excitation of two regions with positive and negative charges, characterized by opposite electric vectors for the each semi-space with respect to the metallic plane, as presented in Fig. 7a.

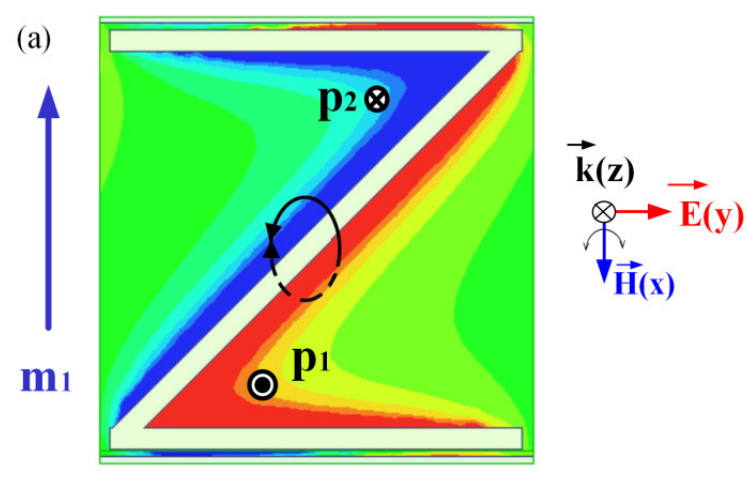

@4.7GHz

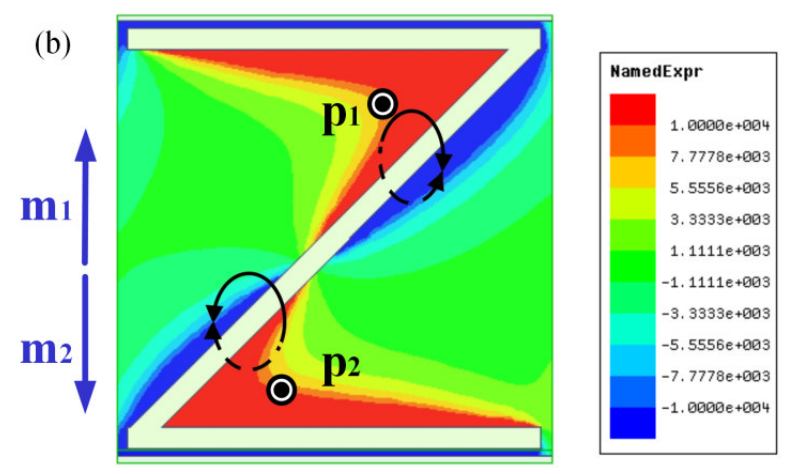

(a) $12.9 \mathrm{GHz}$

Figure 7: Calculated charge distribution for complementary $Z$-structure. (a) Fundamental mode corresponding to a magnetic dipole moment. (b) Dark mode corresponding to an electric dipole moment.

As mentioned, the dark mode in the original structure corresponds to the excitation of two co-directional magnetic dipole moments along $z$-axis and two opposite electric moments parallel to $x$-axis, as shown in Fig. 5b. Therefore, the dual behavior is associated with normal electric dipoles 
and two opposite magnetic dipole moments along $x$-axis, as depicted in Fig. 7b. Two co-directed normal electric moments excited on each side of metasurface are matched with the two opposite axial electric fields that form two regions in the corners of the design with identical charges as displayed in Fig. 7b. Owing to symmetry matching, the contribution of the incident electric field is enhanced by that of the magnetic component of incident field. Consequently, the dark mode in complementary $Z$ structure is characterized by strong electric response with reduced magnetic dipole moment.

It should be noted that dark mode for complementary structure also provides significant increase of quality factor $\left(Q_{\text {dark }}=39\right)$ compared to that of the fundamental resonance with $Q_{\text {fund }}=1.8$, as it can be observed from spectral results shown in Fig. 6. The deviation from the original $Z$, where $Q_{\text {fund }}=0.85$ and $Q_{\text {dark }}=29$, results from less absorption in the substrate due to less uncovered dielectric surface.

\section{Experimental validation}

In order to validate the presented results experimentally, prototypes of $Z$-shaped and complementary $Z$ metasurface are fabricated using printed circuit board technology. Total sample size is $35 \times 35$ cells on a $210 \mathrm{~mm} \times 210 \mathrm{~mm}$ dielectric substrate. The material parameters of the substrate and the geometrical dimensions used for the experimental validation are the same as in numerical simulations. Microwave transmission measurements based on the experimental setup described in [17] have been performed on the fabricated prototypes in an anechoic chamber using an Agilent 8722ES network analyzer and two wideband horn antennas. Phase referencing and normalization have been performed in transmission by removing the sample from the signal path. Experimental results presented in Figs. $6 \mathrm{e}$ and $6 \mathrm{f}$ are in excellent agreement with numerical simulations.

\section{Conclusions}

In conclusion, we have provided demonstration, both numerically and experimentally, in the microwave domain of the direct dark mode excitation mechanisms through symmetry matching. This provides higher flexibility for metasurfaces engineering and results in great improvement of their spectral performances. By using Babinet's principle dual electromagnetic behavior has been demonstrated, not only for fundamental resonance but also for the dark mode resonance. As a result, steep variations in the spectral response with the considered symmetry matching approach can be achieved either in transmission or reflection depending on the type of metasurfaces used. Moreover, the efficient magnetic or electric response can be achieved by engineering metallic structures or complementary designs. The proposed approach entirely relies on the structure's geometry and presents important advantages for multispectral sensor applications.

\section{Acknowledgements}

E. Bochkova acknowledges her Ph.D. scholarship from the French Ministry of Higher Education and Research.

\section{References}

[1] S. E. Harris, "Electromagnetically induced transparency," Phys. Today 50, 36-42 (1997).

[2] M. F. Yanik, W. Suh, Z. Wang, and S. Fan, "Stopping light in a waveguide with an all-optical analog of Electromagnetically Induced Transparency," Phys. Rev. Lett. 93, 233903 (2004).

[3] Q. Xu, S. Sandhu, M. L. Povinelli, J. Shakya, S. Fan, and M. Lipson, "Experimental realization of an on-chip all-optical analogue to electromagnetically induced transparency," Phys. Rev. Lett. 96, 123901 (2006).

[4] R. W. Boyd and D. J. Gauthier, "Photonics: Transparency on an optical chip," Nature 441, 701 (2006).

[5] S. Zhang, D. A. Genov, Y. Wang, M. Liu, and X. Zhang "Plasmon-induced transparency in metamaterials,” Phys. Rev. Lett. 101, 047401 (2008).

[6] B. Luk'yanchuk, N. I. Zheludev, S. A. Maier, N. J. Halas, P. Nordlander, H. Giessen, and T. C. Chong, "The Fano resonance in plasmonic nanostructures and metamaterials," Nat. Mater. 9, 707 (2010).

[7] B. Gallinet and O. J. F. Martin, "Refractive index sensing with subradiant modes: a framework to reduce losses in plasmonic nanostructures," ACS Nano 7, 6978 (2013).

[8] B. Gallinet and O. J. F. Martin, "Influence of Electromagnetic Interactions on the Line Shape of Plasmonic Fano Resonances," ACS Nano 11, 89999008 (2011).

[9] Falcone, F. et al., 2004. Babinet Principle Applied to the Design of Metasurfaces and Metamaterials. Physical Review Letters, 93.

[10] Guo, Y. et al., 2012. Electromagnetically induced transparency (EIT)-like transmission in side-coupled complementary split-ring resonators. Optics Express, 20, p.24348.

[11] Jia, Y.-P. et al., 2014. Complementary chiral metasurface with strong broadband optical activity and enhanced transmission. Applied Physics Letters, 104, p.011108.

[12] V. A. Fedotov, M. Rose, S. L. Prosvirnin, N. Papasimakis, and N. I. Zheludev, "Sharp trapped-mode resonances in planar metamaterials with a broken structural symmetry," Phys. Rev. Lett. 99, 147401 (2007).

[13] C. Forestiere, L. Dal Negro, and G. Miano, "Theory of coupled plasmon modes and Fano-like resonances in subwavelength metal structures," Phys. Rev. B 88, 155411 (2013).

[14] A. Lovera, B. Gallinet, P. Nordlander, and O. J. F. Martin, "Mechanisms of Fano resonances in coupled plasmonic systems," ACS Nano 7, 4527 (2013).

[15] B. Hopkins, A. N. Poddubny, A. E. Miroshnichenko, and Y. S. Kivshar, "Revisiting the physics of Fano resonances for nanoparticle oligomers," Phys. Rev. A 88, 053819 (2013).

[16] S. N. Burokur, A. Lupu, A. de Lustrac, "Direct dark mode excitation by symmetry matching of a singleparticle based metasurface," Phys. Rev. B 91, 035104 (2015).

[17] E. Bochkova, S. N. Burokur, A. de Lustrac, A. Lupu, "Direct dark modes excitation in bi-layered 
enantiomeric atoms-based metasurface through symmetry matching," Optics Letters 41, 412-415 (2016).

[18] N. Wongkasem, A. Akyurtlu \& K.A. Marx, 2006. "Group theory based design of isotropic negative refractive index metamaterial". Progress In Electromagnetics Research, 63, pp.295-310

[19] W.J. Padilla, 2007. "Group theoretical description of artificial electromagnetic metamaterials". Optics Express, 15, p.1639.

[20] J.D. Baena, L. Jelinek \& R. Marqués, 2007. “Towards a systematic design of isotropic bulk magnetic metamaterials using the cubic point groups of symmetry". Physical Review B, 76.

[21] C.M. Reinke et al., 2011. "Group-theory approach to tailored electromagnetic properties of metamaterials: An inverse-problem solution". Physical Review E, 83.

[22] I. Sersic, M.A. van de Haar, F.B. Arango \& A.F. Koenderink, 2012. "Ubiquity of Optical Activity in Planar Metamaterial Scatterers". Physical Review Letters, 108.

[23] ANSYS HFSS (High Frequency Structure Simulator), version 17 (2016).

[24] Jackson, J.D., 1998. Classical Electrodynamics. 